\title{
Aprovechamiento faunístico durante fines del Holoceno medio y el Holoceno tardío al sur de la cuenca superior del río Santa Cruz: el caso de Río Bote 1
}

\section{Victoria Fiel ${ }^{*}$}

Fecha de defensa: 17 de diciembre de 2020

Directora: Dra. Nora V. Franco

Codirectora: Dra. Laura Marchionni

Jurados: Dres. Karen Borrazzo y Diego D. Rindel

En las últimas décadas los análisis zooarqueológicos en Patagonia meridional se han centrado en el estudio de las estrategias de aprovechamiento faunístico adoptadas por los grupos humanos. Dicha problemática ha sido abordada por varios autores desde diferentes perspectivas teóricometodológicas, teniendo en cuenta las condiciones tanto geográficas como climático-ambientales y sociales (De Nigris, 2004; Marchionni, 2013; Mengoni Goñalons, 1999; Otaola, 2008; Rindel, 2009; entre otros). Particularmente, al sur de la cuenca superior del río Santa Cruz hacia fines del Holoceno medio y Holoceno tardío se produjeron fluctuaciones climáticas (Franco, Borrero, Brook y Mancini, 2018; Franco, Brook, Mancini y Vetrisano, 2016), las cuales afectaron la estructura de los recursos y probablemente, en consecuencia, el comportamiento de los grupos humanos y sus modos de subsistencia, como lo sugieren los resultados obtenidos por Otaola (2008) en el sitio Chorrillo Malo 2, ubicado en el ecotono bosque-estepa al sur de Lago Roca. Además, hacia fines del Holoceno medio y comienzos del Holoceno tardío los antecedentes arqueológicos sugieren el inicio de la ocupación efectiva en el área (Franco, 2004, Franco et al., 2018), en conjunto con el registro de cambios culturales, tales como el cambio de la técnica de talla de hojas a una de talla Levallois, sensu Boëda (1993) y la aparición de entierros humanos bajo rocas (Franco, Cirigliano y Ambrústolo, 2011; Franco, Galimani, Moranga y Borrero, 2017; Franco et al., 2018). En consecuencia, se ha considerado que la variación ambiental podría haber influido en las estrategias de apropiación y procesamiento de presas por parte de los grupos humanos, así como en los procesos de ocupación del área (Borrero, 1994-1995; Franco, 2002; Franco et al., 2018; Franco y Borrero, 2003; Otaola, 2008; entre otros).

En base a estos antecedentes, el tema central de esta tesis -enmarcada dentro de los proyectos: UBACyT 20020130100664BA: "Comportamiento y circulación de cazadores-recolectores en la cuenca del río Santa Cruz" y National Geographic N 8397-08: "New information about the peopling of the Upper Santa Cruz basin (southern Patagonia, Argentina) and of the role of rock shelters in the area: the site

* Departamento de Ciencias Antropológicas, Facultad de Filosofía y Letras, Universidad de Buenos Aires, Puan 480, $3^{\circ}$ piso (CP C1406CQJ), Ciudad Autónoma de Buenos Aires, Argentina. E-mail: mvictoriafiel@gmail.com
Río Bote 1"- fue contribuir al análisis de la subsistencia de los grupos cazadores-recolectores que frecuentaron el sur de la cuenca superior del río Santa Cruz (Patagonia, Argentina), desde una perspectiva arqueofaunística. El objetivo central fue examinar el aprovechamiento de la fauna, con especial énfasis en el procesamiento del guanaco por parte de los grupos cazadores-recolectores que habitaron el sito Río Bote 1 entre 4200 y 350 años AP. A su vez, se compararon los resultados aquí obtenidos con aquéllos producidos por Otaola (2008) para el sitio Chorrillo Malo 2, ubicado a $70 \mathrm{~km}$ al Oeste de Río Bote 1 , con el fin generar una visión de mayor alcance respecto de las estrategias de aprovechamiento del guanaco en la cuenca superior del río Santa Cruz.

El análisis comparativo de la fauna se realizó teniendo en cuenta las características paleoclimáticas, los antecedentes arqueológicos del área y la presencia de entierros en el sitio. Para ello se dividió la muestra en cuatro bloques temporales: 1) Primer bloque: entre 4200 y 4100 años AP; 2) Segundo bloque: entre 4100 y 3900 años AP; 3) Tercer bloque: entre 3800 y 3600 años AP; 4) Cuarto bloque con un único fechado de 350 años AP (Franco et al., 2016, 2018).

Para evaluar los procesos de formación del sitio se analizó todo el conjunto arqueofaunístico; no obstante, en lo relativo al estudio de la subsistencia humana, se hizo especial énfasis en el procesamiento del guanaco. La asignación de los restos óseos a un taxón específico fue alcanzada únicamente cuando los especímenes contenían rasgos diagnósticos a nivel anatómico (Mengoni Goñalons, 1999) y, en caso contrario, fueron determinados a niveles más inclusivos como género, familia, orden y clase (Salemme, Miotti y Tonni, 1988). Para analizar la abundancia anatómica y taxonómica del conjunto se calcularon medidas derivadas del número de especímenes identificados por taxón (Klein y Cruz Uribe, 1984; Lyman, 2008), contemplando lateralidad y estado de fusión ósea (Kaufmann, 2009). Seguidamente se realizó un examen de las modificaciones óseas que pudieron afectar la estructura de los conjuntos (Gifford-González, 1981, 2018; Marean, 1995, entre otros). Se realizó un estudio exhaustivo de los indicadores de actividad antrópica, analizándose tanto los tipos de huellas como la fragmentación ósea en relación con los recursos que cada porción anatómica puede brindar y los tipos de fractura (Behrensmeyer, 1978; Gifford-González, 2018; Johnson, 1985). Para evaluar la representación anatómica del guanaco se utilizó el índice de densidad mineral ósea propuesto por Elkin (1995) en conjunto con distintos índices de anatomía económica (Borrero, 1990; Lyman, 1992; Mengoni Goñalons, 1996; Mengoni Goñalons y De Nigris, 1999; Morín, 2007). Por último, para 
analizar la intensificación del aprovechamiento de los recursos del guanaco se incluyó la propuesta de Wolverton (2002), la cual evalúa el procesamiento de los animales relacionando el índice de fragmentación y el porcentaje de porciones enteras de cada elemento.

En lo que hace a los resultados obtenidos, en primer lugar, los análisis permitieron concluir que los procesos tafonómicos que afectaron al sitio variaron en relación con las condiciones ambientales. Los conjuntos pertenecientes al primer y segundo bloque se encuentran en mejor estado de preservación. Esto se vincula, principalmente, con una mitigación de los efectos de la diagénesis y la biodegradación de los restos arqueofaunísticos debido a la elevada presencia de carbonato de calcio circulando entre los sedimentos y a condiciones estables a nivel sedimentológico. En el tercer período, por otro lado, los procesos tafonómicos vinculados con el cavado de las estructuras de entierros humanos habrían resultado en un conjunto más fragmentado postdepositacionalmente. Finalmente, el conjunto óseo del cuarto bloque temporal se habría visto más expuesto a las condiciones ambientales, debido a un cambio en la morfología del sitio por una modificación del curso del río (Franco et al., 2018), resultando en un conjunto mediado por las condiciones meteóricas y diagenéticas.

Los resultados también indican que Río Bote 1 presenta evidencias claras de aprovechamiento y procesamiento faunístico orientado a la subsistencia entre ca. 4200 y 4100 años. Durante este período se registró el aprovechamiento de la carcasa del guanaco de manera extensiva. Además, de manera concordante con lo esperado para momentos de condiciones climáticas favorables para la ocupación humana y baja densidad demográfica, no se registra un procesamiento intensivo de dicho animal. Este panorama difiere con lo que ocurre en Chorrillo Malo 2 para fines del Holoceno medio, cuando las estrategias utilizadas dan cuenta de una selección imparcial de selección de unidades anatómicas del guanaco (Otaola y Franco, 2008).

Entre ca. 4100-3900 años AP, en cambio, la evidencia en Río Bote 1 sugiere tanto un uso extenso de la carcasa del guanaco como un procesamiento intensivo de aquellas unidades que brindan mayor cantidad de tuétano y grasas óseas. Debido a que para este momento los antecedentes sugieren la ocupación efectiva de estos espacios y no se registran variaciones ambientales, estos patrones de procesamiento de fauna parecen indicar la existencia de stress nutricional en los grupos cazadores-recolectores producto de un posible aumento de su demografía. Por otra parte, el registro de instrumentos óseos en este bloque, indica la existencia de una mayor variación en el aprovechamiento del guanaco con respecto al primer bloque temporal.

Hacia ca. 3800-3600 años AP, el registro faunístico sugiere el desarrollo de una estrategia de intensificación sólo sobre aquellas unidades anatómicas del guanaco que aún conservan recursos intraóseos cuando dichos animales sufren stress nutricional. Por primera vez en la secuencia, el registro arqueofaunístico da cuenta de una disminución en la extensión del aprovechamiento del guanaco y sus falanges no parecen haber sido procesadas para obtener sus nutrientes intraóseos. Dado que este período es inmediatamente posterior a la erupción del volcán Aguilera y se caracteriza por condiciones de mayor aridez, se esperaba que en Río Bote 1 se mantuviera un patrón de extensificación sobre el esqueleto del guanaco e intensificación sobre aquellos elementos con alto y bajo contenido de grasas. Sin embargo, los resultados no apoyan esta idea. En este sentido, la evidencia faunística sugiere que Río Bote 1, en estos momentos, podría estar presentando un contexto vinculado al ámbito funerario. Por el contrario, para estos momentos Chorrillo Malo 2 continúa mostrando evidencias de aprovechamiento faunístico vinculadas a la subsistencia humana. La presencia de grupos cazadoresrecolectores frecuentando Chorrillo Malo 2 entre ca. 4300/3800 a 2500 años AP, con patrones de procesamiento de fauna completamente diferentes a Río Bote 1 entre ca. 3800-3600 años AP es consistente con la idea de la complementariedad de estos espacios (Franco et al., 2018).

Finalmente, la evidencia faunística fechada en ca. 350 años AP refuerza la idea presentada en los antecedentes de que se habría dado un cambio en la forma de utilización del área o la presencia de nuevos grupos humanos, usando de manera diferente estos espacios, como lo sugiere la evidencia genética de poblaciones humanas de este sector de Patagonia, además de cambios en la tecnología lítica, en las materias primas utilizadas y en los patrones funerarios (Franco et al., 2017 , 2018). No obstante, en el caso de Río Bote 1 la evidencia de procesos posdepositacionales y de mezcla en este último período no permite realizar una interpretación más completa sobre las actividades llevadas a cabo en el sitio.

\section{Referencias citadas:}

»Behrensmeyer, A. K. (1978). Taphonomic and ecology information from bone weathering. Paleobiology, 4, 150162.

» Boëda, E. (1993). Le débitage discoid et le débitage Levallois recurrent centripete. Bulletin de la Societé Préhistorique Francaise, 90(5), 392-404.

" Borrero, L. A. (1990). Fuego-patagonian bone assemblages and the problem of communal guanaco hunting. En L.B. Davis y B. O. K. Reeves (Eds.), Hunters of the Recent Past (pp. 373-99). London: Unwin Hyman.

»Borrero, L. A. (1994-95). Arqueología de la Patagonia. Palimpsesto, 4, 9-69.

»De Nigris, M. E. (2004). El consumo en grupos cazadores recolectores. Un ejemplo zooarqueológico de Patagonia meridional. Buenos Aires: Sociedad Argentina de Arqueología. 
"Elkin, D. C. (1995). Volume density of South American camelids skeletal parts. International Journal of Osteoarchaeology, 5, 29-37.

»Franco, N. V. (2002). Estrategias de utilización de recursos líticos en la cuenca superior del río Santa Cruz. (Tesis doctoral inédita). Universidad de Buenos Aires, Argentina.

"Franco, N. V. (2004). La organización tecnológica y el uso de escalas espaciales amplias. El caso del sur y oeste de Lago Argentino. En A. Acosta, D. Loponte, y M. Ramos (Eds.), Temas de Arqueología, Análisis Lítico (pp. 101-144). Luján: Universidad Nacional de Luján.

"Franco, N. V. y Borrero, L. A. (2003). Chorrillo Malo 2: initial peopling of the Upper Santa Cruz Basin. En R. Bonnichsen, L. Miotti, M. Salemme y N. Flegenheimer (Eds.), Where the South Winds Blow. Ancient Evidences of Paleo South Americans (pp. 149-152). Texas: A\&M University Press.

»Franco, N. V., Borrero, L. A., Brook, G. A. y Mancini, M. V. (2018). Changes in the Technological Organization and Human Use of Space in Southern Patagonia (Argentina) During the Late Holocene. En E. Robinson y F. Sellet (Eds.), Lithic Technological Organization and Paleoenvironmental Change: Global and Diachronic Perspectives (pp. 301-320). Cham: Springer.

»Franco, N. V., Brook, G., Mancini, M. V. y Vetrisano, L. (2016). Changes in lithic technology and environment in southern continental Patagonia: The Chico and Santa Cruz River basins. Quaternary International, 422, 57-65.

» Franco, N.V., Cirigliano, N. y Ambrústolo, P. (2011). Semejanzas en tecnologías, diseños y prácticas funerarias al sur de la cuenca superior del río Santa Cruz: algunos ejemplos correspondientes al Holoceno tardío. En L. A. Borrero y K. Borrazzo (Eds.) Bosques, Montañas y Cazadores: Investigaciones Arqueológicas en Patagonia Meridional (pp. 155-178). Buenos Aires: Instituto Multidisciplinario de Historia y Ciencias Humanas (IMHICIHU), CONICET.

"Franco, N. V., Galimany, J., Moraga, M. y Borrero, L.A. (2017). La integración de información cultural y genética en el sur de la cuenca superior del río Santa Cruz: primeros resultados. Trabajo presentado en Décimas Jornadas de Arqueología de la Patagonia. Puerto Madryn, Argentina.

" Gifford-González, D. P. (1981). Taphonomy and Paleoecology: A critical review of Archaeology's sister disciplines. Advances in Archaeological Method and Theory, 4, 365-438.

" Gifford-González, D. P. (2018). An Introduction to Zooarchaeology. Springer, International Publishing AG.

» Johnson, E. (1985). Current developments in bone technology. Advances in Archaeological Method and Theory, 8, 157-235.

》 Kaufmann, C.A. (2009). Estructura de Edad y Sexo en Guanaco. Estudios Actualísticos y Arqueológicos en Pampa y Patagonia. Buenos Aires: Sociedad Argentina de Antropología.

» Klein, R. G. y Cruz Uribe, K. (1984). The Analysis of Animal Bones from Archaeological Sites. Chicago: The University of Chicago Press.

» Lyman, R. L. (1992). Anatomical considerations of utility curves in zooarchaeology. Journal of Arehaeological Science, 19, 7-22.

» Lyman, R. L. (2008). Quantitative Paleozoology. Cambridge: Cambridge University Press.

» Marchionni, L. (2013). Comparación de las distintas historias tafonómicas en los conjuntos zooarqueológicos provenientes de la meseta central de la provincia de Santa Cruz (Tesis doctoral inedita). Universidad Nacional de La Plata, Argentina.

" Marean, C. W. (1995). Of taphonomy and zooarchaeology. Evolutionary Anthropology, 4(2), 64-72.

» Mengoni Goñalons, G. (1996). La domesticación de los camélidos sudamericanos y su anatomía económica. En D. C. Elkin, C. M. Madero, G. Mengoni Goñalons, D. E. Olivera, M. C. Reigadas y H. D. Yacobaccio (Eds.), Zooarqueología de Camélidos (pp. 33-45). Buenos Aires: Grupo Zooarqueología de Camélidos.

" Mengoni Goñalons, G. (1999). Cazadores de Guanaco de la Estepa Patagónica. Buenos Aires: Sociedad Argentina de Antropología.

» Mengoni Goñalons, G. y De Nigris, M. (1999). Procesamiento de huesos largos de guanaco en Cerro Los Indios 1 (Santa Cruz). Soplando en el viento. Actas de las Terceras Jornadas de Arqueología de la Patagonia (pp. 461-475). Neuquén y Buenos Aires: Universidad del Comahue e Instituto Nacional de Antropología y Pensamiento Latinoamericano.

» Morín, E. (2007). Fat composition, skeletal part selection, and Nunamiut decision- making. Journal of Archaeological Science, 34, 69-82.

»Otaola, C. (2008). Explotación faunística durante el Holoceno medio y tardío en el sitio Chorrillo Malo 2, Provincia de Santa Cruz, Argentina. ¿Variaciones o continuidades? (Tesis de licenciatura inédita). Universidad de Buenos Aires, Argentina.

»Otaola, C. y Franco, N. V. (2008). Procesamiento y consumo de guanaco en el sitio Chorrillo Malo 2, provincia de Santa Cruz, Argentina. Magallania, 36(2), 205-219.

»Rindel, D. D. (2009). Patrones de procesamiento faunístico durante el Holoceno medio y tardío en el sitio Alero Destacamento Guardaparque (Parque Nacional Perito Moreno, Provincia de Santa Cruz, Argentina) (Tesis de Licenciatura inédita). Universidad de Buenos Aires, Argentina.

»Salemme, M., Miotti, L. y Tonni, E. (1988). La determinación sistemática de los mamíferos en el análisis arqueofaunístico. En R. Ratto y A. F. Haber (Eds.) De Procesos, Contextos y otros Huesos (pp. 65-75). Buenos Aires: Facultad de Filosofía y Letras, Universidad Nacional de Buenos Aires.

"Wolverton, S. (2002). NISP:MNE and \%WHOLE in analysis of prehistoric carcass exploitation. North American Archaeologist, 23(2), 85-100. 